Ebisu Ebisu

Études japonaises Études japonaises

57 | 2020

Les architectes de l'ère Heisei (1989-2019). Rôles, statuts, pratiques et productions

\title{
Homo habitans. Le musée de Teshima
}

\section{François Bizet}

\section{(2) OpenEdition \\ Journals}

Édition électronique

URL : http://journals.openedition.org/ebisu/5411

DOI : 10.4000/ebisu.5411

ISSN : 2189-1893

Éditeur

Institut français de recherche sur le Japon à la Maison franco-japonaise (UMIFRE 19 MEAE-CNRS)

Édition imprimée

Date de publication : 15 décembre 2020

Pagination : 371-394

ISSN : $1340-3656$

\section{Référence électronique}

François Bizet, «Homo habitans. Le musée de Teshima », Ebisu [En ligne], 57 | 2020, mis en ligne le 20 décembre 2020, consulté le 29 mars 2021. URL : http://journals.openedition.org/ebisu/5411 ; DOI : https://doi.org/10.4000/ebisu.5411 


\section{Homo habitans \\ Le musée de Teshima}

François BIZET**

Pour Geneviève

Le musée de Teshima* 豊島, dans la mer intérieure de Seto, mesure soixante mètres de long sur quarante mètres de large. Hauteur : 4670 millimètres, au plus élevé de la structure. Voilà pour les dimensions. C'est un cosmos principalement constitué de vide. Les visiteurs y entrent par une petite porte en alcôve, que l'architecte du lieu, Nishizawa Ryūe* 西沢立衛 (né en 1966), a voulue aussi étroite que le lui permettait la législation - au compte-goutte donc : "Après vous... ", " Je vous en prie... ", et le dos légèrement courbé.

Sitôt passé le sas, la colonne vertébrale se retend et reconquiert sa position verticale. Malgré le peu d'élévation de la voûte, elle trouve ici un habitat à son échelle. La forme du crâne s'adapte sans tarder à sa nouvelle demeure, à l'amplitude de cette coque de béton sans suture apparente, et

La première version de ce texte a reçu le $1^{\text {er }}$ prix du concours d'écriture Henry Jacques

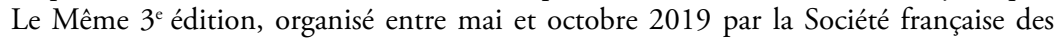
architectes.

* Les mots suivis d'un astérisque renvoient au lexique situé à la fin du dossier.

** Libraire à Paris pendant dix ans, puis lecteur dans les universités d'Ankara et de Yeditepe (Istanbul) de 1993 à 2004, il est maintenant maître de conférences à l'université de Tokyo et écrivain. Pour une liste de ses publications, voir : https://francois-bizet.com. 
percée de deux orbites énormes où abondent le bleu du ciel et les nuances de la verdure. Une sorte d'ermitage, un couvent sans cellules, une nouvelle génération de cloître, tout en rondeurs, où l'on viendrait pour une retraite temporaire reprendre ses esprits, où chacun serait simultanément accueilli et élargi : vous, vers l'ouverture ouest, moi, du côté est. Mais seule la distribution de l'ombre et de la lumière décide des flux.

Quelque chose me dit que nous sommes ici enfin chez nous. Il a fallu, pour y accéder, prendre le train de Tokyo jusqu'à Okayama, et de là transiter en ferry par Naoshima 直島, l'île voisine, jusqu'à celle de Teshima. Un autobus conduit du port au milieu des rizières qui dominent la baie, mais la distance se parcourt aussi à pied. Après un long périple, nous voilà donc de retour. À l'abri : cela saute aux yeux, et comme enveloppés - mais, paradoxe tout aussi manifeste, sans toit ni murs porteurs, et qui plus est, en plein courant d'air. Littéralement : expulsés en dedans. Au contact de la vastitude - on entre ici comme dans une mosquée, sans chaussures, et en été, pieds nus -, et à la seule vue de ces surfaces lisses et fuyantes, de cet horizon uniformément gris perle, quelque chose en nous se ressaisit et se dilate. Nous sommes remis au centre, et dès que remis en jeu, projetés, ballottés dans toutes les dimensions du volume. Au choix : météore ou pelote. Personne, dans cette géométrie rien moins qu'angulaire, qui ne rebondisse à loisir, au hasard, pour rien, pour voir. J'atterris, vous atterrissez, nous atterrissons sans fin. L'exploration prend des allures diverses : qui une allègre déambulation, qui la lenteur contemplative. Un autre, la position assise. Ou couchée, un sourire aux lèvres. Les corps se meuvent, s'arrêtent, se lèvent, se considèrent au passage - que pourraient-ils faire d'autre? -, et si l'on s'éloigne, c'est pour revenir sur ses pas, si l'on s'agrège, c'est pour aussitôt se désagréger tous azimuts, sans plan aucun, aléatoirement. On ne se frôle qu'à distance. On s'aimante, on s'esquive dans un mouvement sans cesse renouvelé par le flux de nouveaux arrivants, introduits à mesure des départs. Des milliers de communautés transitoires se forment et se déforment ici chaque jour, selon ces lois locales, variables, de gravitation. Parfois, un enfant prend la tangente. Là, la densité se fait nulle. Plus loin, on assiste à un alignement, ou comme à une éclaircie. Et pas un heurt. Tous autant que nous sommes, nous nous sentons évoluer dans un espace sans pièges ni mots d'ordre, pacifié, amical, voué au nonchaloir et aux murmures. L'envers d'une salle des pas perdus, aux heures de pointe. Le milieu, remis à l'endroit. Et chacun, avec une exquise courtoisie, à sa place. 
Jamais je n'ai eu cette sensation que j'éprouve ici de toucher terre, comme au terme d'une longue chute. De me rétablir, mais en douceur. Tout autour, dans le silence froissé de vents marins, finit d'ailleurs de se poser la soie encore légèrement bombée d'un vaste parachute.

Sensation d'être nu, comme au premier jour, à découvert, au milieu des arbres.

Mais de musée, point...

L'humain ici rejouerait plutôt, au moment même de retrouver l'humble compagnie de la courbe terrestre ou d'un piaillement de mouette, de la pluie sur l'herbe ou des insectes dans la lumière de midi, son lignage animal. Nous avons été recueillis dans des mains techniciennes, et parmi les plus expertes car comment faire tenir ce voile à peine soulevé de terre, ce palais, si modeste soit-il, sans la moindre colonne? Pourtant, cette architecture qui nous offre un temps son hospitalité n'est ni triomphale ni conquérante. Elle ne sépare pas les mondes. Au contraire, elle les superpose. Sous la bosse parfaitement concave, à ras du sol, j'inspecte mon terrier. Je me fais taupe. Puis j'entre en léthargie. Plus tard, une immense couveuse dissout mes limites corporelles. Et si au cours de la rêverie le bulbe involue en coquille, ce n'est pas de celles rigoureusement chantournées que les vagues, en contrebas, rejettent sur les rives pour la fascination et l'édification des hommes. Cette coquille-là, à si faible distance maintenant de mes mains qui pourraient en palper ou en imprimer la paroi, est encore molle de la chaleur des organes qui l'ont conçue, et tremble entre deux eaux génésiques. Elle rappelle plutôt l'œuf dans sa matrice que le nautile adulte, charpenté jusque dans les derniers replis de ses labyrinthes. Tout en elle regimbe contre l'envoûtement exercé par la forme et son achèvement : l'indifférence à l'élan du matériau, son refus des hauteurs comme de la claustration, sa porosité. Son immatérialité d'écume.

Laissons cette bulle percoler jusqu’à notre conscience claire, jusqu’à éclosion...

Comment ne pas se souvenir ici que le Japon est un pays volcanique, en ébullition permanente?

Que le mont Fuji n'a pas toujours eu cet éclat diamantin? Qu'il a, lui aussi, comme tout le monde, commencé très bas, quasiment au niveau de la mer, par un balbutiement de projectiles en fusion et de bruits de forge. 
Je me demande si nous ne sommes pas en train d'assister au retour, sur cette petite île cernée par la suractivité industrielle, d'un modèle primitif du bâti.

À l'affleurement d'un âge des Fluides.

Du Protozoaire.

Du Têtard...

Il nous faudrait descendre encore... Nous rapprocher de la surface.

Ma première visite au musée de Teshima ${ }^{1}$ remonte à 2011. Ce jour-là, je n'ai pas eu trop de deux minutes d'attentive progression vers le centre, bercé par la douceur du dôme, pour deviner que l'essentiel se jouait aussi par terre, à mes pieds. À deux ou trois mètres de moi, en effet, dans un coin inférieur de mon champ visuel, il y a eu comme un remuement. Quelque chose avait fui, à mon approche peut-être, avec la vélocité d'un scolopendre. C'est ainsi en tout cas que j'ai aussitôt interprété le minuscule événement optique qui venait à la fois de se produire et de m'échapper, tout en m'avisant qu'il était peu probable qu'un mille-pattes ait pu surgir d'un plancher de béton et s'y replier à son gré, car à l'endroit de l'agitation, l'œil une fois accommodé, il n'y avait aucune trace de présence animale. J'ai regardé à la ronde, un peu troublé par l'hallucination. D'autres visiteurs autour de moi scrutaient le sol, contournaient des flaques d'eau plus ou moins grandes que je n'avais pas remarquées d'abord tant elles se fondaient dans l'étendue. Peut-être attendaient-ils comme moi que se reproduise le même phénomène. Il n'était pas difficile de comprendre que les grandes flaques (ce mot est d'ailleurs impropre car une flaque a besoin pour se former d'une dépression du sol : celles-là au contraire débordaient le plan, comme une ampoule la peau, ou sur le mur un miroir convexe) étaient régulièrement alimentées par les plus petites. Le mouvement furtif capté tout à l'heure n'était donc autre que le ruissellement de l'une dans l'autre. Mais d'où pouvait bien venir que l'eau prenne tout à coup la décision de s'écouler? La pente du sol était pour ainsi dire nulle : il fallait nécessairement que cette étrange nappe phréatique atteigne une sorte de masse critique qui la force à pencher du

1. Appellation officielle : Teshima Art Museum. https://benesse-artsite.jp/en/art/teshima-artmuseum.html (dernière consultation le 4 mai 2018). 
côté le plus favorable aux méandres. Et puisque l'eau ne venait pas du ciel, sans nuage ce jour-là, elle devait nécessairement sourdre des profondeurs.

Voyons ça de plus près.

Le sol du musée, produit d'un si soigneux polissage qu'on dirait presque une pièce de velours, est percé, comme une branche de corail, de trous microscopiques. Il y en a partout. Un ici, un autre là. Où l'on enfoncerait tout juste une épingle. Le recensement est impossible mais nous oblige à redoubler de politesse envers cette base d'un nouveau genre, animée, sensitive - excitable? Nous marchions sans le savoir sur une éponge, au-dessus d'immenses coulisses souterraines... Quelle machinerie! D'autant de sources invisibles perle un liquide maîtrisé à la seconde, au terme d'une circulation qu'on imagine très alambiquée à travers pompes et tuyaux, probablement commandés par ordinateur. L'ingéniosité de ce théâtre hydraulique est indéniable, ainsi que sa puissance visuelle : la lentille d'eau qui point des sous-sols a la forme même de la coupole qui l'accueille, celle d'une goutte de rosée, étincelante de condensation, telle que je l'ai vue hier matin sur les feuilles planes des nénuphars du jardin de Ritsurin, au nord de la grande île de Shikoku : juste en face à vol d'oiseau. Rappel du large!... Dématérialisation, rematérialisation... Nous sommes bien ici entre terre et ciel, dans cet espace éminemment plastique, acentré, nébuleux et turbulent, espèce de membrane réactive saturée d'oxygène et d'hydrogène - dans l'intervalle, si bien figuré par les deux valves de béton. Nous sommes ici où la vie s'organise, entre les roches les plus fraîches de l'écorce et la basse atmosphère, dans l'étroitesse de cette bande habitable où prospère l'unité des vivants et de leur biotope, et que les géosciences appellent : la zone critique. Nous y sommes, ou plutôt : nous en sommes. Parties prenantes du milieu, nous attendons la naissance de l'élément des éléments. Son émergence silencieuse et son écho en nous. On a pris place et, posté près d'un des orifices, on assiste le miracle organique.

Il a lieu.

Ce que vous teniez pour un simple agrégat de sable et de bitume, transpire. L'eau ici ne jaillit pas, elle distille avec une extrême lenteur. C'est à proprement parler une retenue. Oh! rien de commun avec le bavardage voluptueux de grandes eaux royales, ou d'une fontaine de Trevi... Aucun rapport frontal, qu'imposerait le monument, ou la statuaire - chevaux marins, nymphes et tritons. Le Japon est une terre de sources thermales : des eaux brûlantes sont puisées dans les fractures, mais sitôt rendues à la 
surface, réparties dans de vastes bassins, parfois même en terrasses, pour l'enveloppement collectif, étale, horizontal des corps. Eaux profondes, lustrales. Il y a une volupté tranquille de l'immersion ${ }^{2}$. Le musée de Teshima n’a pas oublié ce principe si populaire du onsen 温泉, qui est aussi celui des rizières avoisinantes. Les visiteurs y sont moins accueillis qu'immédiatement mis dans le bain, et introduits de plain-pied dans l'œuvre à voir, contenu et contenant. Aucune barrière muséographique, donc, entre le regardant et le regardé, le moins de distance possible et au contraire, la possibilité partout de l'échange épidermique. La terre comme le corps sont sudoripares. La perméabilité est totale, et vous êtes, au milieu de ce réseau hydrographique embryonnaire - comme un poisson dans l'eau, ou un courant tempéré, ou comme ces spectateurs de haut rang à qui l'on consentait le droit d'être assis sur la scène parmi les comédiens, dans le décor - participant de l'exsurgence et de l'écoulement, de l'évaporation et des précipitations.

Espace gouttelant, fusions et diffusions. "Leau ne se tient pas à distance ", écrit Gilles Clément lorsqu'il parle des nuages. "Elle nous enveloppe, nous pénètre ainsi qu'elle le fait pour tout organisme vivant et tout objet inerte ${ }^{3}$."

Notre perle entre-temps a augmenté en volume. Elle a doublé, décuplé sa masse, irriguée par des millions d'années de poussées tectoniques et des milliards d'années de préhistoire intersidérale. Tant qu'elle maintiendra en équilibre l'empilement de ses liaisons atomiques, j'aurai la fixité d'une bête à l'affût. J'attendrai l'instant où, les forces ainsi agencées excédant la capacité de résistance de l'édifice, elles iront s'épancher au petit bonheur. À Teshima comme ailleurs dans l'Archipel, impossible d'oublier que la terre est régulièrement secouée de séismes, dont les plus violents résolvent des tensions colossales, accumulées dans le temps long. La libération d'énergie prend ici la forme d'un éclair liquide : un lézard scintillant file doux vers un tout petit lac, lui aussi tout près de débonder et de laisser libre cours au trop-plein, et de pièce en pièce jusqu'à la grande flaque ouest, en aval, sous le soleil qui en fait son ordinaire. Le sol est ainsi parcouru d'innombrables départs de feu qui forment un réseau sans cartographie possible de

2. Lire à ce sujet Mizubayashi Akira 水林章, "Dans le bain japonais ", Critique, $\mathrm{n}^{\circ}$ 428-429, 1983, p. 5-15.

3. Clément Gilles, Nuages, Paris, Bayard, coll. «Le rayon des curiosités », 2004, p. 11. 
failles fantômes, d'oueds immédiatement asséchés. Même le lit ne laisse aucune empreinte. Les ruisseaux ne coulent pas, ils glissent. Ils s'écrivent et s'effacent à mesure. Ils ont l'existence éphémère d'un pur tracé.

Nom de l'artiste : Naitō Rei 内藤礼. Titre de l'œuvre : Matrix4.

Imaginons un musée où les visiteurs se pencheraient au-dessus des œuvres d'art comme un enfant au-dessus d'une colonne de fourmis, les enjamberaient, s'allongeraient tout près d'une aquarelle, ou d'une série in progress de sanguines. Ou bien rêvasseraient entre deux encres - celles, gigantesques, de Kakinuma Kōji 柿沼康二? -, et à deux doigts, à le toucher, du calligraphe.

Liquéfaction des frontières.

Le musée de Teshima n'est pas vide, mais de l'œuvre qu'il contient peuton dire qu'elle est exposée? Selon un étrange renversement, c'est moi qui me retrouve, par l'architecture même, exposé aux forces extérieures et universelles, au cycle entier de l'eau mis en œuvre par Naitō Rei. Or Matrix, comme l'indique son titre, renvoie aussi aux forces du dedans, gestation ou genèse. Impossible d'opérer une distinction. On est pris dans un lieu imprenable, entre-deux, ici et là - où? On ne reconnaît plus rien. Ça, un musée? Il faudrait accepter de se défaire des définitions, des normes, de l'histoire, tout oublier, remonter aux sources, jusqu'à l'immémorial, et pourquoi pas au beau milieu de ces Muses qui en leur temps élisaient domicile en pleine nature, préférablement dans les grottes, et dont Pausanias avance qu'une première génération, avant que Zeus et Mnémosyne ne s'en mêlent, était née de l'accouplement d'Ouranos avec Gaïa : entre terre et ciel ${ }^{5}$.

Est-ce en raison d'une vague similitude géographique que j'ai glissé si facilement de la mer intérieure de Seto à la Méditerranée? Que je me vois

4. On trouvera une chronologie et un commentaire des œuvres de Naitō Rei dans Transphère, $\mathrm{n}^{\circ} 3$, "Rei Naito. Émotions de croire ", Maison de la Culture du Japon à Paris \& Les Presses du Réel, 2017, p. 25-32.

5. Pausanias, Description de la Grèce, tome V, livre IX, $\$ 29$, trad. M. Clavier, Paris, Société Royale Académique des Sciences, 1821, p. 159. 
tout d'un coup transporté de l'autre côté du globe, en Béotie, sur les hauteurs de l'Hélicon, dans le sanctuaire de Calliope et de ses sœurs, à deux pas de la fontaine Hippocrène qui leur était consacrée et où Hésiode dit qu'elles lavaient " leur corps délicat ${ }^{6}$ " : au cœur du museion donc - bien avant toutefois sa métamorphose en musée, en monument. En institution. Je pourrais m'amuser de ces coïncidences et passer à autre chose, aux choses sérieuses, mais quelque chose justement me dit que l'essentiel est là, dans cette soudaine dénudation du spectateur que je est devenu, de ce pur regard que plusieurs siècles de mutations muséologiques, des Wunderkammern de la Renaissance au White Cube moderne ${ }^{7}$, à travers des kilomètres de galeries de la Tate et du Prado, des Offices et de l'Ermitage, ont appris à orienter, à aiguiser.

Ici, l'œil n'a plus de guide. Il tâtonne à ciel ouvert.

$\mathrm{Nu}$ comme un ver au sortir de sa motte.

Plus que renversant en effet, le musée de Teshima est déboussolant. Les pôles habituels ayant disparu, la flèche ne sait plus où se fixer. Rien, sur ces murs où l'accrochage est impensable, qui puisse assouvir cette impérieuse pulsion qu'on appelle scopique. Et peut-on même encore parler, du creux de cette vague prodigieusement ourlée, d'un mur? D'un appui? Tout semble agencé pour la dérive, pour l'errance périphérique, pour la divagation sans objet, pour le roulement indéfini. Aucun plan, aucun angle. Lidée de cadre a été refondue en une immense pliure ondoyante. Même les fenêtres se sont amollies. Tout déborde : le contenant dans le contenu, en un mutuel et incessant ressourcement des formes, et le dehors vers l'intérieur, d'où l'œil est constamment invité à s'exorbiter. Le contraire donc de la chambre, du studiolo, du cabinet, ou de la "galerie idéale " de Brian O’Doherty : "Quelque chose de la sacralité de l'église, du formalisme de la salle d'audience, de la mystique du laboratoire expérimental s'associe au design chic pour produire cette chose unique : une chambre esthétique.

6. Hésiode, Théogonie, trad. Leconte de Lisle, Paris, Alphonse Lemerre éditeur, 1869, p. 3.

7. Lire les travaux de Falguières Patricia, Les Chambres des merveilles, Paris, Bayard, coll. "Le rayon des curiosités ", 2003 et "À plus d'un titre ", Préface à O’Doherty Brian, White Cube. L'espace de la galerie et son idéologie, trad. C. Vasseur, Zurich, JRP/ Ringier, 2008 [1973-1981], p. 5-32. 
À l'intérieur de cette chambre, le champ magnétique est si puissant que s'il en sort, l'art peut déchoir jusqu'à un statut séculier ${ }^{8}$."

Qu'est-ce qui pourrait bien déchoir dans cet antre plein de trous où tout est voué à l'écoulement, à la circulation perpétuelle? Un tel risque de déclassement laisse indifférente la vie cyclique d'une molécule d'eau, véritable électron libre de l'art. L'œuvre de Naitō Rei ne pouvait trouver refuge que dans l'ajouré et l'espacement; elle a aussi trouvé son site : $34^{\circ} 29^{\prime}$ $23^{\prime \prime}$ de latitude nord et $134^{\circ} 05^{\prime} 28^{\prime}$ ' de longitude est, que le rayonnement solaire la conduit à évacuer sans relâche. Et sans laisser d'adresse.

Art erratique...

Tout le contraire, de ce point de vue, des œuvres bien acclimatées du musée Chichū (Chichū bijutsukan 地中美術館) de Naoshima ${ }^{9}$, à quelques milles d'ici - une heure environ par la mer. L'insularité de l'espace d'exposition et la sanctuarisation de l'art n'y sont sans doute nulle part plus manifestes que dans la salle qui renferme quelques beaux Nymphéas de Claude Monet.

Traduction de chichī : « dans la terre ».

De loin, en effet, puis de si près que l'on s'approche de la rive, on ne voit rien d'autre qu'un promontoire recouvert d'une végétation épaisse. Point de musée. Mais sitôt les hauteurs atteintes, sous un soleil de plomb, par une route en forte pente, le visiteur est pris en charge, coaché pourrait-on dire par un personnel formé au tourisme de masse. Excepté le hall d'accueil qui délivre les billets et les informations, rien ne dénonce encore la présence d'une construction qu'on a voulu la plus discrète possible afin de préserver le paysage : entre ce premier seuil et l'entrée proprement dite, un long sentier bordé d'une flore rivulaire, multicolore, rappelle les promenades de Giverny. Puis, tout au bout, qui le prolonge, un corridor gris comme une lame de couteau. L'architecte, Andō Tadao* 安藤忠雄 (né en 1941), a tranché dans la lumière et vous conduit, d'oblique en oblique, vers les profondeurs, à travers les antichambres, entre les parois de béton verni,

8. Ibid., p. 36 .

9. Appellation officielle: Chichu Art Museum. https://benesse-artsite.jp/en/art/ chichu.html (dernière consultation le 4 mai 2018). 
luisant dans la pénombre, jusqu'aux abords d'une pièce brillamment blanchâtre, un peu brumeuse, qu'on aperçoit de loin, comme une promesse. Contrairement à ce que l'œil éprouve d'abord, c'est bien une lumière zénithale, diffusée d'une verrière mais amortie par un plafond factice, qui nimbe les cinq Nymphéas, encadrés dans un marbre blanc de Thassos, et met en valeur les sept cent mille tout petits pavés de marbre de Carrare (celui de la Pietà de Michel-Ange, précise la notice), qui composent le sol.

Les cryptes, parce que cernées d'épaisses murailles, sont par définition obscures. Celle-là est merveilleusement, c'est-à-dire surnaturellement éclairée. Une telle qualité laiteuse, presque moussue, d'un jour abstrait et sans origine, relayée sous la plante des pieds par la tiédeur du parterre minéral, perpétue avant tout le projet originel, étymologique de la scénographie : " art de représenter en perspective ${ }^{10}$ ". Tout ici a pour fonction d'isoler du tumulte extérieur, d'instaurer une clôture, une capsule spatio-temporelle bien hermétique - bref, une bulle à l'intérieur de laquelle le chef-d'œuvre apparaît en majesté, central, éternellement émergent du fond de sa substance immaculée et nourricière, et face à la foule sans cesse renouvelée des adorateurs, éternellement sur le point de fuir ${ }^{11}$.

Je ne vais pas bouder mon plaisir : ces Nymphéas sont admirablement mis en scène. On pourrait même oser, dans la logique également spéculative de Fukutake Sōichirō 福武總一郎 (né en 1945), mécène des lieux : de manière optimale. Pas plus d'une quinzaine de visiteurs en même temps, le temps d'une brève communion. Je quitte la salle comblé mais quelque peu songeur au moment d'embrasser, dans un dernier coup d'œil, le clair reposoir de nouveau serti d'ombre et la longue file d'attente des pèlerins déchaussés.

Ces centaines de milliers de visiteurs qui explorent chaque année, en quelques jours, les trois îles du Benesse Art Site Naoshima (Benesse āto saito

10. Grand Robert de la langue française. P. Falguières parle d'une "révolution de la scénographie ": "Entre 1480 et 1513, à Urbino, à Rome, la perspective centrée, inventée par et pour les peintres, impose son pouvoir de cadrage à l'aire de jeu scénique " (Falguières Patricia, "Le Teatro Olimpico de Palladio et la refondation du théâtre dans l'Europe du XVI ${ }^{\mathrm{e}}$ siècle ", conférence, université de Tokyo, 2017).

11. Sur l'insularité de l'espace muséal, lire Sloterdijk Peter, Écumes. Sphères III, trad. O. Mannoni, coll. « Pluriel », 2014 [2003], p. 297-298. 
Naoshima ベネッセアートサイト直島 ${ }^{12}$ ) gérées par l'héritier de la Fukutake Publishing, aujourd'hui Benesse Holdings (100\% de Berlitz International, Inc.) - à savoir : la minuscule Inujima 犬島 (et son ancienne raffinerie de cuivre), Teshima, et surtout Naoshima, le fleuron de l'entreprise de réhabilitation, par l'art, des territoires désertifiés -, sont invités à loger chez l'habitant (minshuku 民宿), dans les pensions locales (ryokan 旅館), ou encore dans des yourtes plutôt rustiques, aménagées sur la plage, en contrebas de la Benesse House. Cette maison mère abrite la collection familiale: Twombly, Yanagi [Muneyoshi] 柳宗悦, Warhol, Giacometti, Nauman, Sugimoto [Hiroshi] 杉本博司, Rauschenberg, Hockney, César, Francis... Mais elle offre également aux amateurs que laisserait insatisfaits un parcours standard, la possibilité d'être hébergés dans l'enceinte même du musée, ouvert pour eux toute la nuit, dans les chambres d'un véritable hôtel haut de gamme qui en sont autant d'extensions à la carte, personnalisées : ainsi telle suite possède une pièce de Thomas Ruff, telle twin des photographies de Bernd et Hille Becher, cette suite avec vue une œuvre de Jennifer Bartlett. Quant aux « suites ovales ", elles vous permettent de vous endormir sous la garde d'une œuvre de Richard Long ou David Tremlett, à moins que ne vous tente le minimalisme d'Imi Knoebel ou de Sol LeWitt.

Pour ce qui me concerne, j'ai réservé une des yourtes du rivage. Pendant la journée, elles sont inhabitables. On y suffoque de chaleur. La température baisse subitement au coucher du soleil mais un ventilateur est indispensable jusqu'au matin. J'ai passé trois nuits dans ce décor de steppe, à peine isolé du dehors par une toile tendue sur un treillis de bois circulaire, à tourner et me retourner sous un toit peint de formes symboliques. Trois nuits d'insomnie, au niveau de la mer, avant de continuer vers le sud, vers Kyūshū.

12. Toujours selon l'appellation officielle, ce qui tendrait à confirmer l'ambition internationale du projet. https://benesse-artsite.jp/en/ (dernière consultation le 4 mai 2018). 


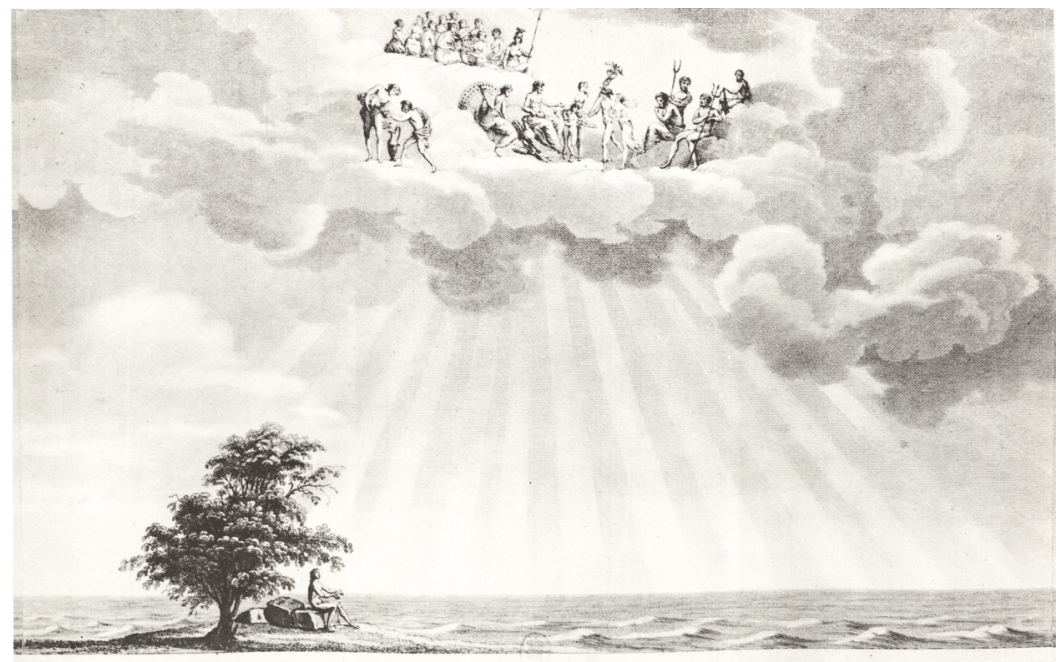

L'Abri du pauvre, gravure de Claude-Nicolas Ledoux, 1804.

Retour à Teshima.

Au point de rosée.

Ce sont peut-être les mots qui m'ont traversé l'esprit, au passage de la porte basse, lorsque j'ai refait le voyage quelques années plus tard, pour revoir, pour me confirmer à moi-même que tout cela avait bien eu lieu. C'est en tout cas par cette formule empruntée à la météorologie que mon second séjour me revient.

Que reste-t-il du musée, lorsque le principe de collection a été à ce point liquidé? À Teshima, l'œuvre, unique, fait corps avec l'architecture, qui du coup perd son statut multiséculaire d'écrin : Panthéon d'Hadrien, utopies sphériques d'Étienne-Louis Boullée, Crystal Palace ${ }^{13}$, Louvre d'Abu Dhabi... À ce régime spatial aujourd'hui dominant, où les œuvres sont susceptibles d'être transportées d'une boîte à l'autre, Nishizawa Ryūe et

13. Sur cette réalisation de Joseph Paxton, lire Sloterdijk Peter, op. cit., p. 304-305, et Sloterdijk Peter, Le Palais de cristal. À l'intérieur du capitalisme planétaire, trad. O. Mannoni, Paris, Maren Sell Éditeurs, 2006 [2005]. C'est d'ailleurs dans cet essai que j'ai trouvé la formule " homo habitans» (p. 41). 
Naitō Rei substituent une sorte d'opération chimique : émulsion, condensation, précipitation. Les parois se retournent, s'invaginent, rejoignent la qualité primordiale de leur hôte : la flaccidité. Quelque chose se fige en masse plus ou moins solide, comme ne peut mieux dire l'étymologie. Plus ou moins : c'est l'oscillation qui m'intéresse. Un appareil homogène, onctueux, coulé d'une seule traite - celui de ces laitages qu'on désigne sous le nom de blanc-manger - a remplacé dirait-on l'ancienne maçonnerie, toute de blocs équarris et de jointures. Oui, c'est bien à cela que nous assistons, au tremblement d'une conglomération nouvelle, d'une solution matérielle et esthétique où il n'est plus possible de distinguer l'habitant de l'habitacle, la perle de la coquille. Équilibre précaire, en reconquête constante. Comme si notre cosmos, déposé de fraîche date, possédait à la fois les propriétés des fluides et du mortier; comme s'il gardait, dans sa charpente intime, le souvenir de l'instabilité du cratère, et le nomadisme du cumulonimbus. En vérité, il penche pour l'un et pour l'autre. Une bulle, peut-être, mais prise à un moment critique de sa formation ou de sa vaporisation.

C'est cet ensemble que je veux appeler le génie du lieu.

À quoi sert d'enfouir les bâtiments? Pourquoi les traiter en parties honteuses? Il y a là, finalement, une manière d'alibi. Ne suffisait-il pas de tirer parti d'une efflorescence, à même la surface, une légère surrection du sol, sous la forme d'abord d'un simple tas, une espèce de tumulus de base vaguement elliptique, tumescence presque naturelle qu'on augmenterait, qu'on armerait dans un deuxième temps d'un châssis d'acier propre à recueillir des milliers de litres d'un béton tout spécialement conçu, sur une épaisseur de vingt-cinq centimètres, lequel parement mettrait cinq semaines à sécher avant que ne commence le long travail d'excavation du moule de terre?

Exactement ce qui a été fait.

Il ne restait plus alors qu'à laisser venir. Laisser s'exprimer le socle, le laisser jaser et gazouiller de lui-même, et dialoguer avec la flore, les météores, les arthropodes et les bipèdes. Le laisser devenir une composante de la biosphère. Un microclimat.

Nulle pudeur de bon ton, donc. Le musée de Teshima ne respecte pas le paysage, il l'épouse. C'est ce que j'aime ici : que la forme démoulée doive moins au projet et à l'intention bâtisseuse qu'aux lignes et aux dynamiques locales. Que le manteau serve de patron.

Ce que j'aime encore : l'extrême légèreté de l'habillage. Certes, je me tiens debout sous une chape, mais comme la veste du couturier Yamamoto 
Yōji 山本耀司 dont Wim Wenders fit l'éloge dans Tokyo-ga, elle ne donne pas l'impression de peser. Elle m'enrobe sans m'entraver, à la lettre : elle m'environne. Ma toute première intuition se confirme donc: il s'agit bien d'un abri, à condition de se souvenir que ce nom dérivé de l'ancien abrier, issu du bas latin par modification de l'apricari classique, est lui-même tout droit venu d'apricus : " exposé à la chaleur du soleil ». Le dictionnaire parle à ce point d'une origine obscure, ce qui n'est pas sans piquant pour un terme qu'à l'époque on rapprochait d'aperire, "ouvrir ", entendu comme " lieu ouvert au soleil ». Drôle de mot en effet, s'il abrite en ses commencements le sens contraire de celui développé plus tard par l'usage, qui est de s'épargner les désagréments d'une soumission aux intempéries du dehors. Étrange fossile... De quel changement de sensibilité au monde cette volte-face sémantique est-elle le signe? Qu'il ait fallu se protéger est dans l'ordre des choses mais qu' "abri » ait justement été choisi pour le signifier semble témoigner de l'affaiblissement d'un rapport immédiat, immanent au monde des phénomènes célestes - imperceptible révolution, sans commune mesure mais non sans résonance secrète avec le projet de mise à distance et d'arraisonnement de la Nature, qui est celui de la modernité.

Ici comme partout, le monde se dérobe. Le cosmos se retire. Du fait d'une intensification continue de la lumière artificielle, un tiers de la population mondiale est aujourd'hui privé d'une expérience sensible constitutive de notre condition terrestre : la vision au-dessus de nos têtes de la Voie lactée. D'abri, la planète est devenu gisement, réserve exploitable, objet de placements et de rapport. Nous évoluons désormais hors-sol, dans la terre promise du global. Et dans la boucle d'un paradoxe, car c'est au moment où nous mesurons l'ampleur du démariage, par la révélation quasi quotidienne des déprédations irrémédiables de l'homme sur le milieu, que nous sommes tentés d'inscrire notre nom dans l'inconnu qui s'ouvre: Anthropocène. Or, parallèlement aux débats indispensables quant à la manière de nommer la période géologique nouvelle où nous nous sommes nous-mêmes jetés ${ }^{14}$, on ne compte plus les appels pressants, presque comminatoires, à reformuler nos manières d'habiter le monde, à réinventer notre habitat. Et ce dans des disciplines diverses : l'anthropologie, à travers Philippe Descola, qui appelle

14. Fressoz Jean-Baptiste \& Bonneuil Chritophe, L'Événement Anthropocène. La Terre, l'histoire et nous, Paris, Seuil, " Points ", 2013, p. 119-315. 
à " une véritable écologie politique, une cosmopolitique ${ }^{15}$ "; la géographie, par la voix d'Augustin Berque, lorsqu'il suggère de " recosmiser la terre ${ }^{16}$ " par la reconsidération du ciel; ou la philosophie, par celle d'Emanuele Coccia : "Nous ne sommes pas des habitants de la terre; nous habitons l'atmosphère $^{17}$ ".

Qu'est-ce que cela veut dire?

De l'atmosphère, nous savons qu'elle a été en partie constituée par l'activité photosynthétique des arbres et de l'herbe. En tant qu'interface cosmique primordiale, en tant que faiseur de monde, le végétal est donc le modèle privilégié d'une compénétrabilité des milieux et des êtres vivants : si les premiers informent les seconds, ils ne cessent également d'être façonnés par eux. La primauté de la loi de rétroaction, «fluidification » ou « compénétration ${ }^{18}$ " que défendait déjà James E. Lovelock ${ }^{19}$, et avant lui Vladimir Vernadski ${ }^{20}$, implique selon Coccia la dissolution de nos catégories fondamentales. Ainsi du climat, désormais défini en termes de non-dualité : "Dans tout climat la relation entre contenu et contenant est constamment réversible: ce qui est lieu devient contenu, ce qui est contenu devient lieu $^{21}$ ». Ainsi encore de notre terre, qui ne saurait être considérée comme une surface impassible, pur réceptacle indépendant de la vie qui y prolifère et s'y adapte, et pas même comme un lieu: "Le monde n'est pas un lieu; il est l'état d'immersion de toute chose dans toute autre chose ${ }^{22}$ ". Autrement dit, et à rebours de ce que tend à imposer une certaine doxa humaniste, nous n'avons jamais été séparés. La vie, de sa forme la plus archaïque à la plus complexe, est avant tout présence à la fois perméable et diffuse. Impossible, par conséquent, de continuer à parler d'une sortie des eaux,

15. Descola Philippe, "Humain, trop humain?", in Beau Rémi \& Larrère Catherine (dir.), Penser l'Anthropocène, Paris, Les Presses de Sciences Po, 2018, p. 33.

16. Berque Augustin, Recosmiser la terre, Paris, Éditions B2, 2018.

17. Coccia Emanuele, La Vie des plantes. Une métaphysique du mélange, Paris, Bibliothèque Rivages, 2016, p. 51.

18. Ibid., p. 53 et 54 .

19. Lovelock James Ephraim 1979, Gä̈a, A New Look at Life on Earth, Oxford, Oxford University Press (traduction française en 1993).

20. Vernadski Vladimir, La Biosphère, Librairie Félix Alcan, "Nouvelle collection scientifique", 1929 [1926] (NB : Aucune mention du traducteur).

21. Coccia Emanuele, op. cit., p. 41.

22. Ibid., p. 89. 
mutation évolutionnaire capitale du Silurien, première d'une longue série mythique de dégagements jusqu'à Homo erectus, sinon à la faire suivre d'une entrée en atmosphère. Sapiens, tout extérieur à la nature qu'il s'affirme, est aujourd'hui comme toujours un poisson dans l'eau, un être en relation.

Faire un pas dans le musée de Teshima, c'est donc immédiatement éprouver ceci : "Le ciel n'est pas ce qui est en haut. Le ciel est partout ${ }^{23}$ ". La verticalité vacille. Nous vivons à l'abri des nuages et des rayons solaires, dont nous sommes une émanation, une concrétion certes très sophistiquée, mais une expression comme une autre. Il est bien entendu que "ciel » ici désigne tout autre chose que cette altitude à la conquête de laquelle, dans les multiples centres de la conurbation pacifique, on lance des tours interminables. Prenons une des dernières en date, Tokyo Sky Tree : ses 634 mètres ne pourront jamais que conforter les visiteurs dans l'icarisme et le délire de maîtrise panoramique de l'espèce. Les corps, eux, du haut du belvédère vitré et climatisé, demeurent étrangers aux frôlements des turbulences, concentrés qu'ils sont dans le seul globe oculaire (et son extension mécanique : la longue-vue), en rotation au milieu de son empire. Aux antipodes d'une telle hubris, prométhéenne, désormais planétaire, à quoi peuvent donc bien prétendre les deux orbites énucléées de la coque de Teshima, sinon à nous refaire des organes, et à hauteur d'arbre, un tout nouveau régime sensoriel? À nous aboucher au cosmos, comme y encourageait déjà la poésie, par la voix de Francis Ponge ${ }^{24}$ ? Et nous ouvrir, mais autrement : aux flux, à tous les flux, ceux d'en haut et ceux d'en bas, pour nous y fondre et nous en sentir traversés... Pour nous y réveiller, après des millénaires d'hominisation et d'arrachements à la gangue, en êtres de passage - capillaires, volatiles...

Je suis assis depuis près d'une demi-heure dans une des périphéries de la voûte, les yeux tournés vers les nuages d'automne. Combien d'autres corps, pendant ce temps, ont-ils discrètement quitté les lieux, combien y ont-ils été introduits? Ces communautés éphémères de Teshima sont changeantes, instables, elles n'ont pas la compacité de celles que réunissent les théâtres autour d'un seul foyer, avec lesquelles je les ai un temps confondues. Mais elles diffèrent aussi de celles, éparses, des musées classiques, en ce sens que

23. Ibid., p. 119.

24. Ponge Francis, "Le monde muet est notre seule patrie ", Méthodes, Euvres complètes I, Gallimard, "Bibliothèque de la Pléiade », 1999 [1961], p. 630. 
pour la première fois peut-être dans l'histoire de l'art, un public tout entier se découvre une affinité matérielle, une identité de nature avec l'œuvre exposée, et peut-être même de contenance, puisque indifféremment, tantôt selon les lois de l'hydrosphère, tantôt selon l'affluence (haute saison ou saison creuse), et l'humeur de chacun, les masses en présence se font et se défont, s'étirent, se dissipent, se succèdent sans s'abolir, s'échangent de part et d'autre de la paroi - au compte-goutte.

Je suis resté assis là longtemps, à me perdre à la recherche d'un nouveau contour. À la fin de l'opération, étais-je si loin du " pauvre » entrevu par l'architecte Claude-Nicolas Ledoux lorsqu'il entreprit de colliger, au seuil du XIX ${ }^{e}$ siècle, tous les projets de sa vie? Au beau milieu des gravures représentant des temples, des barrières d'octroi, des palais de justice, des théâtres et des ateliers, des guinguettes et des églises, des ponts, des forges à canon préindustrielles, des salines panoptiques, insolite parmi ces visions d'une géométrie à la fois primitive et grandiose - un abri, nu, désert, « la maison du pauvre ", toute d'éléments en formation (condensation et développement) et de mouvements tourbillonnaires : "ondes ", " molécules ", " amalgames ${ }^{25}$ ".

Architecture première, atmosphérique...

Un abri, donc, voire un refuge, et pourquoi pas une demeure... mais à l'air libre.

Pour éloigné qu'il paraisse des canons architecturaux de l'Archipel, le musée de Teshima illustre pleinement cette constante structurelle du " goût japonais pour le caractère ouvert de l'espace ${ }^{26}$ ». Selon Sendai Shōichirō 千代章一郎 en effet, l'habitat domestique traditionnel ne reconnaît de mur (kabe 壁) qu'exempté de la fonction de portance, partout assurée par le bois des poteaux et des poutres. Si sa solidité apparente ne tient dans certains cas que par l'enduit apposé sur un torchis végétal, le mur consiste le plus souvent en un accessoire léger, fragile, amovible (papier ou tissu), passeur de lumière et parfois même invisible, car virtuel, comme l'indique

25. Ledoux Claude-Nicolas, L'Architecture considérée sous le rapport de l'art, des moeurs et de la législation, vol. I, Paris, Imprimerie H.L. Perronneau, Planche 33, 1804, p. 104.

26. Sendai Shōichirō, "Le mur ", in Bonnin Philippe, Nishida Masatsugu \& Inaga Shigemi (dir.), Vocabulaire de la spatialité japonaise, Paris, CNRS Éditions, 2014, p. 210. 
le mot kabeshiro 壁代 qui renvoie au seul emplacement d'une cloison. Conséquence : "la délimitation intérieur/extérieur demeure très vague, voire flottante $e^{27}$ ", impression renforcée par la multiplication des espaces intermédiaires, dont le plus remarquable est sans doute ce qu'on appelle ici : engawa 縁側, large galerie de transition assimilable à une véranda sans vitrages, avec laquelle le déambulatoire de Teshima, malgré la modernité du matériau, offre aussi une parenté profonde. En somme, tous les éléments consacrés de la maison japonaise semblent avoir été repris pour être refondus autrement, d'un seul tenant. Tous, y compris le toit, yane 屋根 dont Sendai rappelle que l'origine mêle deux sens contradictoires : "demeure précaire, temporaire ", et " racine, chose stable, essentielle, implantée dans la terre ". "Une sorte d'oxymore ${ }^{28}$ ", donc. Mais il précise surtout que c'est le toit, non le mur, qui constitue dans l'habitat nippon ancien la toute première unité d'organisation de l'espace. C'est le toit qui définit la limite du dedans. Et c'est encore lui qui, jusqu'au début au moins du $\mathrm{Xx}^{\mathrm{e}}$ siècle, chez Tanizaki Jun.ichirō 谷崎潤一郎 par exemple, est invoqué pour figurer l'acte d'habiter : " [...] lorsque nous entreprenons la construction de nos demeures, avant toute chose nous déployons ce toit, ainsi qu'un parasol qui détermine au sol un périmètre protégé du soleil, puis dans cette pénombre nous disposons la maison ${ }^{29}$ ". Chercher l'ombre, mais à ciel ouvert; trouver à s'acclimater, en se sachant sur le départ : voilà l'utopie de Teshima. On n'habite pas ici, au sens hérité du fréquentatif de l'avoir latin - on ne peut faire que passer.

Les concepteurs du lieu ne sont d'ailleurs pas les seuls au Japon à élaborer de nouvelles formes architecturales en accord avec ce principe, mais je devrais plutôt dire : à les rêver. Et qu'importe même qu'elles soient irréalisables. Qu'importe que les maquettes minuscules d'Ishigami Jun.ya* 石上純也 (né en 1974), faites de matériaux pauvres, papier, bois clair et fil de fer, que ses bâtis aériens, lisses, simplissimes jusqu'à l'immatérialité, n'appellent aucun maître d'œuvre. C'est justement là, dans ce retrait de l'imagination pure, allégée, libérée de toute contrainte d'ordre physique

27. Ibid., p. 210.

28. Sendai Shōichirō, "Le toit", in op. cit., p. 531.

29. Tanizaki Jun.ichirō 1977 [1933], Éloge de l'ombre, trad. R. Sieffert, Aurillac, Presses orientalistes de France, p. 50. 
et du despotisme de la faisabilité, de la possibilité même, que le désir de renouvellement peut s'exprimer avec le plus de vigueur :

Un sentier sur la mer.

Un atelier comme une forêt.

Un thêâtre comme une montagne.

La maison du vent.

La maison du ciel bleu.

La maison de la pluie.

La maison du vent et de la pluie.

Architecture en roue libre... Jusqu’à la démesure :

Maison géante. Ici, l'architecture n'est pas un abri isolant l'homme du monde extérieur, elle est conçue pour créer un nouvel environnement naturel. Au-delà d'une certaine dimension, le bâtiment peut engendrer des nuages, faire naitre la neige et la pluie, créer des rivières et des forêts. En accroissant démesurément l'échelle du bâtiment, l'intérieur devient l'extérieur. La limite n'est plus liée à la partition des murs, du sol et du toit, mais à l'écart d'échelle entre ce qui se définit comme intérieur ou extérieur $^{30}$.

Le musée de Teshima me semble relever de cet impossible-là : un projet fou qui aurait miraculeusement trouvé sa voie à travers la matière, tout en évitant l'écueil de la monumentalité, tout en maintenant intactes la frâ̂cheur et la faillibilité inaugurales de l'impulsion. C'est dans un tel espace, infiniment émergent, matriciel, que nous avons été dispersés.

Avec lui aussi que nous composons.

De lui encore qu'il faudra bien finir par nous extraire, pour le laisser derrière nous.

Retour à Tokyo...

Mais où exactement?

La métropole? La mégapole? La mégalopole?

30. Ishigami Junya, How Small? How Vast? How Architecture Grows, trad. Arc en Rêve. Centre d'Architecture de Bordeaux, Ostfildern, Hatje Cantz Verlag, 2014, non paginé. 
"Où atterrir? ", demandait il y a peu Bruno Latour dans un essai sur la crise généralisée qui est la nôtre. "Dans quel monde souhaitons-nous vivre $^{31}$ ? " Les réponses à apporter au climato-scepticisme militant, au caractère désinhibé des dérégulations économique et financière, ainsi qu’à une certaine inertie devant l'augmentation exponentielle des inégalités, sont d'ordre éminemment politique, mais rien n'interdit selon Latour de mêler à cette première expertise celle de l'art. Tout l'encourage au contraire : mieux définis, dans le champ du sensible et de la représentation, les enjeux auront plus de chance de se voir partagés.

Ce livre, comme beaucoup d'autres depuis la parution, dès avant le premier Choc pétrolier, du "Rapport Meadows ${ }^{32}$ ", le répète à l'envi : nous n'avons pas les moyens de la globalisation; la croissance illimitée est incompatible avec la finitude du monde habité. À la fuite éperdue dans « le Global ", à la tentation réactionnaire d'un repli sur "le Local» (illustrée aujourd'hui par toute une déclinaison de postures nationalistes), Latour oppose alors un concept tiers, "le Terrestre ", qui permet d'échapper à cette mortifère alternative : "Alors que le Local est fait pour se différencier en se fermant, le Terrestre est fait pour se différencier en s'ouvrant", premier aperçu contrebalancé par cet autre : «Le Terrestre tient à la terre et au sol, mais il est aussi mondial, en ce sens qu'il ne cadre avec aucune frontière, qu'il déborde toutes les identités ${ }^{33}$ ".

Je me demande si le musée de Teshima, sur sa modeste colline insulaire, n'est pas l'endroit du monde le plus propre à nous faire éprouver, sans s'y restreindre bien entendu, cette nouvelle dimension. Aucun lieu moins naturellement discriminant, à ma connaissance en tout cas, ni plus foncièrement hospitalier. Un très haut lieu, en somme, où rejouer notre lien aux éléments

31. C'est une des questions posées aux étudiants qui suivent le Master d'Expérimentations en Arts Politiques (SPEAP), créé en 2010 par Bruno Latour à Sciences Po. http://blogs.sciences-po.fr/speap/presentation/pourquoi-speap/ (dernière consultation le 16 mai 2018).

32. Meadows Donella, Meadows Dennis \& Randers Jorgen, Les Limites de la croissance dans un monde fini, trad. A. El Kaim, Éditions Rue de l'échiquier, 2012 [1972] (rapport commandé par le Club de Rome).

33. Latour Bruno, Où atterrir? Comment sorienter en politique, Paris, La Découverte, 2017, p. 72. 
et aux autres, à tous les autres, aux vivants de partout. Une arche où pouvoir nous refaire, comme on le dit d'un joueur momentanément à sec. Dans ce sens, nous sommes voués (mais pas condamnés) à revenir à Teshima, à ras de terre, à même l'élément brut, et à y converger, à nous y ressourcer sans fin, mais ce retour-là n'a nullement le sens ordinaire d'un " retour à ". L'anthropologue y insiste: après des siècles d'objectivation de la Nature, au terme d'un long mouvement de "sortie ", c'est la Terre - chtonienne, tellurique, climatique - qui fait retour, c'est «le Terrestre " qui se rappelle à l'humain. Ledoux avait tout loisir, dans "L'Abri du pauvre ", d'invoquer la Nature : réduite, après Galilée et Newton, à une série d'équations et de mouvements réguliers, elle lui apparaissait d'abord comme une étendue à humaniser, un horizon rationalisable. Or il nous revient à nous de découvrir que la Terre a aussi et surtout, dit Latour, " un comportement ", et c'est alors un peu comme si "sous la "nature", le monde réapparaissait ${ }^{34}$ ". Retour de, donc, plutôt que retour à. La rectification pourra-t-elle suffire à faire taire ceux qui se complaisent à fausser le débat en raillant une nostalgie fantasmée de la bougie ou de la terre battue? Même si ce recours à l'intimidation trahit de plus en plus manifestement la caducité de l'idéologie, ce que certains n'hésitent pas à appeler une " mystique de la croissance ${ }^{35}$ ", une telle fausse bravoure semble avoir quelques beaux jours devant elle.

Pendant ce temps, contre vents et marées, le musée de Teshima continuera de remonter des profondeurs. De se soulever de terre. De rejouer le mystère des commencements. Son éclosion à la surface de notre zone critique est un événement inépuisable : tout l'univers faisant irruption dans une courbure inconnue de notre œil, encore et encore, entre deux champs de riz. Quelque chose comme une cosmogenèse de poche.

Comme la naissance, à échelle humaine, d'une galaxie.

Je continuerai quant à moi d'en ressentir les secousses, la vibration, mais à distance.

Il n'est pas dit en effet que j'y retourne jamais.

C'est qu'un tel lieu ne se laisse pas habiter.

34. Latour Bruno, Face à Gaïa. Huit conférences sur le nouveau régime climatique, Paris, Les Empêcheurs de tourner en rond/La Découverte, 2015, p. 82 et 85.

35. Méda Dominique, La Mystique de la croissance. Comment s'en libérer?, Paris, Flammarion, 2019. 
Nous n'y sommes pas chez nous.

Il ne nous accueille pas.

Il nous hante.

J'ai appris récemment qu'il s'en était fallu de peu que l'île ne devînt, à la fin du siècle dernier, une décharge industrielle à ciel ouvert, une plaie au milieu de la mer Intérieure. Mais la mobilisation de la population, des artistes et de leur mécène a eu raison de la logique productiviste, qui engendre autant de biens que de rebuts ${ }^{36}$. On ne peut que se féliciter d'un tel dénouement. Se réjouir que la dévastation ait été stoppée avant d'atteindre le point irréversible. Mais, dans un pays touché par un accident nucléaire majeur, où l'on compte encore des milliers de réfugiés et d'innombrables zones contaminées, désertifiées, cette information laisse comme un goût amer. Combien de territoires remis à flot pour tant d'autres en perdition, combien de paysages sauvés pour toutes les solitudes produites par les conquêtes de l'homme, où Michelet voyait en son temps la destruction définitive, hommes et bêtes et paysages, du " génie du lieu ${ }^{37}$ "?

Tokyo, juin 2018

36. Kagawa Prefecture (dir.), Teshima Waste Treatment Project, 2004 : https://www.pref. kagawa.lg.jp/haitai/teshima/epdf.html (dernière consultation le 14 janvier 2019).

37. Michelet Jules, La Mer, coll. "Folio-Classique", 1983 [1861], p. 261. Je me permets ici de renvoyer à un texte écrit trois ans après la catastrophe de Fukushima : "L'inhabitat" - réflexion sur ce que signifie habiter dans un monde meurtri, ravagé par la menace radioactive. Pendant la rédaction d' "Homo habitans", il m'est venu que les deux textes pourraient être réunis dans un même volume : tête-bêche. C'est ainsi, dans cette pliure de l'espace, que j'aimerais qu'on les imagine. (Bizet François, "L'inhabitat ", in Doumet Christian \& Ferrier Michaël (dir.), Penser (avec) Fukushima, Nantes, Éditions Cécile Defaut, 2016, p. 161-190). 


\section{Bibliographie}

\section{Berque Augustin 2018}

Recosmiser la terre, Paris, Éditions B2.

\section{Bızet François 2016}

«L'inhabitat », in Doumet Christian \&

Ferrier Michaël (dir.), Penser (avec)

Fukushima, Nantes, Éditions Cécile

Defaut: 161-190.

\section{Bonneuil Chritophe \& Fressoz Jean-}

Baptiste 2013

L'Événement Anthropocène. La

Terre, I'histoire et nous, Paris, Seuil,

coll. « Points ».

\section{Clément Gilles 2004}

Nuages, Paris, Bayard, coll. « Le rayon des curiosités».

\section{CoccIA Emanuele 2016}

La Vie des plantes. Une métaphysique du mélange, Paris, Bibliothèque Rivages.

\section{Collectif 2017}

«Rei Naito. Émotions de croire »,

Transphère, $\mathrm{n}^{\circ} 3$, Maison de la Culture du Japon à Paris \& Les Presses du Réel.

\section{Descola Philippe 2018}

« Humain, trop humain? », in Beau

Rémi \& Larrère Catherine (dir.), Penser

I'Anthropocène, Paris, Les Presses de

Sciences Po : 19-35.

Falguières Patricia 2003

Les Chambres des merveilles, Paris, Bayard, «Le rayon des curiosités ».
Falguières Patricia 2008 [1976-1981]

"À plus d'un titre », préface à O'Doherty Brian, White Cube. L'Espace de la galerie et son idéologie, Vasseur Catherine (trad.),

Zurich, JRP/Ringier : 5-32.

FaLguières Patricia 2017

«Le Teatro Olimpico de Palladio et la refondation du théâtre dans l'Europe du xvle siècle ", conférence, université de Tokyo.

\section{HÉSIOdE 1869}

Théogonie, Leconte de Lisle (trad.), Paris, Alphonse Lemerre éditeur.

IsHIGAMI Junya 2014

How Small? How Vast? How

Architecture Grows, Arc en Rêve. Centre d'architecture de Bordeaux (trad.), Ostfildern, Hatje Cantz Verlag.

Kagawa Prefecture (dir.), 2004 Teshima Waste Treatment Project, https:// www.pref.kagawa.lg.jp/haitai/teshima/ epdf.html (dernière consultation le 14 janvier 2019).

\section{Latour Bruno 2015}

Face à Gaïa. Huit conférences sur le nouveau régime climatique, Paris, Les Empêcheurs de tourner en rond/La Découverte.

\section{LATOUR Bruno 2017}

Où atterrir? Comment s'orienter en politique, Paris, La Découverte.

\section{Ledoux Claude-Nicolas 1804}

L'Architecture considérée sous le rapport de l'art, des mours et de la législation, 
vol. I, Paris, Imprimerie H.L. Perronneau, Planche 33.

\section{Lovelock James Ephraim 1979}

Gaïa, A New Look at Life on Earth, Oxford, Oxford University Press.

\section{Meadows Donella, Meadows Dennis \&}

RANDERS Jorgen 2012 [1972]

Les Limites de la croissance dans un monde fini, El Kaim Agnès (trad.), Paris, Éditions Rue de l'échiquier.

MÉda Dominique 2013

La Mystique de la croissance. Comment s'en libérer?, Paris, Flammarion.

Michelet Jules 1983 [1861]

La Mer, Paris, « Folio-Classique ».

MIZUBAYASHI Akira 1983

«Dans le bain japonais », Critique, $n^{\circ} 428-429$ : 5-15.

O'DoherTy Brian 2008 [1976-1981]

White Cube. L'espace de la galerie et son idéologie, Vasseur Catherine (trad.), Zurich, JRP/Ringier.

Pausanias 1821

Description de la Grèce, tome V, livre IX, $\S 29$, M. Clavier (trad.), Paris, Société Royale Académique des Sciences.

Ponge Francis 1999 [1961]

Méthodes, Fuvres complètes I, Paris, Gallimard, «Bibliothèque de la Pléiade ».

\section{Sendal Shōichirō 2014}

"Le mur », « Le toit », in Bonnin

Philippe, Nishida Masatsugu \& Inaga
Shigemi (dir.), Vocabulaire de la spatialité japonaise, Paris, CNRS Éditions : 210-212; 531-534.

SLOTERDIJK Peter 2006 [2005]

Le Palais de cristal. À l'intérieur du capitalisme planétaire, Mannoni Olivier (trad.), Paris, Maren Sell Éditeurs.

\section{SLOTERDIJK Peter 2014 [2013]}

Écumes. Sphères III, Mannoni Olivier (trad.), Paris, « Pluriel ».

TANIZAKI Jun.ichirō 1977 [1933]

Éloge de l'ombre, Sieffert René (trad.), Aurillac, Presses Orientalistes de France.

VernAdSKI Vladimir 1929 [1926]

La Biosphère, Paris, Librairie Félix Alcan, « Nouvelle collection scientifique » (NB : Aucune mention du traducteur). 